\title{
Genome-wide identification of Isw2 chromatin-remodeling targets by localization of a catalytically inactive mutant
}

\author{
Marnie E. Gelbart, ${ }^{1,3}$ Nurjana Bachman, ${ }^{4}$ Jeffrey Delrow, ${ }^{2}$ Jef D. Boeke, ${ }^{4}$ and Toshio Tsukiyama ${ }^{1,5}$ \\ ${ }^{1}$ Division of Basic Sciences and ${ }^{2}$ Genomics Resource, Fred Hutchinson Cancer Research Center, Seattle, Washington 98109 , \\ USA; ${ }^{3}$ Molecular and Cellular Biology Program, University of Washington and Fred Hutchinson Cancer Research Center, \\ Seattle, Washington 98195, USA; ${ }^{4}$ Department of Molecular Biology and Genetics and High Throughput Biology Center, \\ The Johns Hopkins University School of Medicine, Baltimore, Maryland 21205, USA
}

\begin{abstract}
Isw2 ATP-dependent chromatin-remodeling activity is targeted to early meiotic and MATa-specific gene promoters in Saccharomyces cerevisiae. Unexpectedly, preferential cross-linking of wild-type Isw2p was not detected at these loci. Instead, the catalytically inactive Isw2p-K215R mutant is enriched at Isw2 targets, suggesting that Isw2p-K215R, but not wild-type Isw2p, is a sensitive chromatin immunoprecipitation (ChIP) reagent for marking sites of Isw2 activity in vivo. Genome-wide ChIP analyses confirmed this conclusion and identified tRNA genes (tDNAs) as a new class of Isw2 targets. Loss of Isw2p disrupted the periodic pattern of Ty1 integration upstream of tDNAs, but did not affect transcription of tDNAs or the associated Ty1 retrotransposons. In addition to identifying new Isw2 targets, our localization studies have important implications for the mechanism of Isw2 association with chromatin in vivo. Target-specific enrichment of Isw2p-K215R, not wild-type Isw2p, suggests that Isw2 is recruited transiently to remodel chromatin structure at these sites. In contrast, we found no evidence for Isw 2 function at sites preferentially enriched by wild-type Isw2p, leading to our proposal that wild-type Isw2p cross-linking reveals a scanning mode of the complex as it surveys the genome for its targets.
\end{abstract}

[Keywords: Chromatin; ATP-dependent chromatin remodeling; ISWI; genome-wide localization; retrotransposon; tRNA]

Supplemental material is available at http://www.genesdev.org.

Received January 18, 2005; revised version accepted March 3, 2005.

The packaging of DNA into chromatin restricts processes dependent on protein-DNA interactions. Work from numerous laboratories has demonstrated that the "opening" and "closing" of chromatin are important mechanisms to increase or restrict access of proteins to nucleosomal DNA. As a result, the balance between these chromatin states is critical for the regulation of processes such as transcription, DNA replication, and DNA repair. The two major classes of proteins that modulate chromatin structure, histone-modifying enzymes and ATP-dependent chromatin-remodeling factors, have been implicated in the regulation of these processes (for review, see Ehrenhofer-Murray 2004).

ATP-dependent chromatin-remodeling complexes use the energy of ATP hydrolysis to alter histone-DNA con-

${ }^{5}$ Corresponding author.

E-MAIL ttsukiya@fhcrc.org; FAX (206) 667-6497.

Article and publication are at http://www.genesdev.org/cgi/doi/10.1101/ gad.1298905. tacts. They are classified into distinct families according to sequence homology of their conserved ATPase subunit, including SWI/SNF, ISWI, CHD/Mi-2, INO80, and RAD54. Several biochemical activities have been demonstrated for subsets of these complexes, including nucleosome sliding along a DNA template, nucleosome deposition, nucleosome disruption, and histone exchange (for review, see Narlikar et al. 2002; Lusser and Kadonaga 2003; Flaus and Owen-Hughes 2004; Langst and Becker 2004).

Although ISWI complexes were initially identified biochemically, their in vivo functions are beginning to be elucidated. Studies in higher eukaryotes have demonstrated that the regulation of chromatin structure by ISWI complexes impacts a variety of nuclear functions. Specifically, mammalian and Drosophila ISWI complexes have been implicated in the maintenance of chromosome structure (Deuring et al. 2000), DNA replication (Collins et al. 2002; Poot et al. 2004), chromatin assembly (Fyodorov et al. 2004), cohesin loading (Hakimi 
et al. 2002), rDNA silencing (Zhou et al. 2002), and transcription (Deuring et al. 2000; Badenhorst et al. 2002; Yasui et al. 2002).

In contrast, the majority of work on yeast ISWI complexes thus far has focused solely on transcription. The Isw2p ATPase is one of two ISWI homologs in Saccharomyces cerevisiae and forms a two- and four-subunit complex with Itclp and two small histone-fold proteins, Dls1p and Dpb4p (Tsukiyama et al. 1999; Iida and Araki 2004; McConnell et al. 2004). Isw2 represses transcription of early meiotic genes and MATa-specific genes in haploids and MAT $\alpha$ cells, respectively, through the formation of nuclease-inaccessible chromatin structure over the promoters (Goldmark et al. 2000; Ruiz et al. 2003; McConnell et al. 2004). In addition to a-specific genes, Isw2 regulates chromatin structure at other classes of genes repressed by the Tup1-Ssn6 complex (Zhang and Reese 2004). Expression microarray analyses have revealed additional Isw2 transcriptional targets, in particular those genes that Isw2 regulates in a parallel pathway with the histone deacetylase complex, Sin3Rpd3 (Fazzio et al. 2001). Isw2 has also been implicated in transcriptional termination, functioning redundantly with the Isw1 and Chd1 ATP-dependent chromatin-remodeling factors (Alen et al. 2002).

Although all studies to date have demonstrated a role for Isw2 in transcriptional regulation, it is likely that chromatin remodeling by Isw2 impacts other nuclear processes as well. In support of this idea, we and others have observed Isw2-dependent changes in chromatin structure near genes expressed in an Isw2-independent manner (Kent et al. 2001; T.G. Fazzio, M.E. Gelbart, and T. Tsukiyama, unpubl.). Furthermore, Isw2p is sufficiently abundant for one molecule per $2-5 \mathrm{~kb}$ of DNA (T. Tsukiyama, unpubl.), suggesting that there are likely to be additional unknown roles for the complex. However, elucidation of other Isw2 functions has been hindered in part due to the lack of technology to systematically identify its targets in vivo.

Integration of retrotransposons and retroviruses into the genome requires access of a preintegration complex, consisting of integrase, element DNA, and possibly additional factors, to the target DNA, and is therefore likely to be affected by chromatin structure. Integration target selection is strictly regulated, and targeting strategies have evolved to maximize advantage for both the element and the host. For example, HIV integrates into genes that are actively transcribed, particularly those activated by HIV infection, thus maximizing the production of retroviral RNA (Schroder et al. 2002), and this targeting may be mediated by interactions with host proteins such as Inilp, a homolog of the chromatin-remodeling complex protein Snf5p (Kalpana et al. 1994), and LEDGF (Maertens et al. 2003; Cherepanov et al. 2004; Llano et al. 2004). Unlike retroviruses, the entire retrotransposon life cycle is restricted to a single cell, and in small densely packed genomes like that of $S$. cerevisiae, their integration is tightly regulated to avoid disruption of the cellular genome (Craigie 1992; Boeke and Devine 1998). Regulation of integration is effected in part by restricting retrotransposon integration to distinct regions of the genome. The $S$. cerevisiae Ty5 retrotransposon integrates into silent chromatin at telomeres and the MAT loci, ensuring a low level of Ty5 expression postintegration (Zou and Voytas 1997). Both Tyl and the distantly related Ty3 elements use sequences upstream of tRNA genes (tDNAs) and other RNA polymerase (pol) III-transcribed genes as integration sites (for review, see Sandmeyer 2003). Regions upstream of tDNAs are genepoor relative to the rest of the genome (Bolton and Boeke 2003) and thus represent "safe havens" for retrotransposon insertion.

Target site selection is achieved in many systems through tethering mechanisms in which the elementencoded integrase protein interacts with a host protein that guides integration to the target site (for review, see Bushman 2003). Ty5 is targeted to silent chromatin by a physical interaction between the retrotransposon integrase and Sir4p (Zou and Voytas 1997; Xie et al. 2001; Zhu et al. 2003). Similarly, subunits of the pol III preinitiation factors, TFIIIC and TFIIIB, direct Ty3 integration within 1-2 bp of the pol III transcription start site (TSS). This targeting has been reproduced in vitro on naked DNA in a purified system supplemented with the pol III general transcription factors /Chalker and Sandmeyer 1992; Kirchner et al. 1995; Yieh et al. 2000, 2002; Aye et al. 2001). In contrast to the precise integration site selectivity of Ty3, Ty1 exhibits regional specificity, integrating within a $~ 750-b p$ "window" upstream of pol III-transcribed genes (Ji et al. 1993; Devine and Boeke 1996; Bolton and Boeke 2003). Ty1 integration into the permissive region is nonrandom, displaying $\sim 80$-bp periodicity upstream of two tDNA families as well as at single tDNA targets (Bachman et al. 2004). Addition of the pol III machinery to naked DNA during in vitro Ty1 integration reactions did not recapitulate either targeting to the tDNA upstream region or the periodic pattern of integration (S.E. Devine and J.D. Boeke, unpubl.), suggesting that additional factors are required and that the mechanism underlying Tyl integration near pol IIItranscribed genes may be more complex than that for Ty3.

Several studies have implicated chromatin structure in the regulation of Tyl targeting. The 80 -bp periodicity of integration upstream of tDNAs, which have identical coding regions but disparate upstream sequences, suggested that the target preferences are defined by a physical structure (Bachman et al. 2004). In addition, although pol III-transcribed genes are the primary targets of Ty1 integration, less frequent integration events do occur in or near pol II-transcribed genes. Mutations in the chromatin regulators, $R A D 6, H I R 3$, and $C A C 3$, as well as a decrease in histone $\mathrm{H} 2 \mathrm{~A}$ and $\mathrm{H} 2 \mathrm{~B}$ levels have been shown to alter the promoter and/or the orientation bias of Ty1 integration at pol II-transcribed genes (Liebman and Newnam 1993; Rinckel and Garfinkel 1996; Huang et al. 1999). However, these studies did not investigate Ty1 integration at its preferred target sites, nor did they identify chromatin alterations associated with changes in integration pattern. Nothing is known of molecular 
interactions underlying the specificity of Ty1 insertion at its most frequent targets.

In this study, we sought to identify the in vivo targets of the Isw2 chromatin-remodeling complex on a genome-wide scale. We found that the cross-linking pattern of the catalytically inactive Isw2p-K215R mutant, but not wild-type Isw2p, is suitable for this purpose. Genome-wide analysis of Isw2p-K215R localization led to the identification of tDNAs as a new class of Isw2 targets in vivo. Chromatin remodeling by Isw 2 at these loci does not lead to detectable changes in tRNA or Ty1 transcription. Instead, Isw2 is required for the periodic pattern of Ty1 integration upstream of tDNAs, demonstrating that ATP-dependent chromatin remodeling influences integration site selection by a retroelement in vivo. Our ability to identify sites of Isw2 activity from the cross-linking pattern of catalytically inactive Isw $2 \mathrm{p}-\mathrm{K} 215 \mathrm{R}$, but not wild-type Isw2p, also reveals features of the mechanism of Isw 2 action in vivo. It suggests that Isw2 activity is required only transiently at its targets and that the remodeled chromatin state is sufficiently stable in the absence of Isw2 to necessitate relatively infrequent rerecruitment of the complex. Since Isw2 activity was not detected at the loci preferentially enriched by wild-type Isw $2 p$, we propose that the cross-linking of wild-type Isw2p primarily detects a basal interaction of Isw2 as it scans chromatin for its targets. Our results exemplify the problems that can arise when using chromatin immunoprecipitation (ChIP) of a wild-type enzyme as a marker for its activity in vivo.

\section{Results}

Wild-type Isw2p is not preferentially enriched at Isw2 targets

Isw2 complex is required to repress a-specific genes in $M A T \alpha$ cells through the formation of a mating-type-specific chromatin structure (Ruiz et al. 2003; McConnell et al. 2004). As shown previously, nucleosome mapping at the STE6 promoter by micrococcal nuclease (MNase) digestion of chromatin followed by indirect end-labeling demonstrated that, in wild-type MAT $\alpha$ cells, the DNA downstream of the $\alpha 2$ operator is packaged into positioned nucleosomes (Fig. 1A, lanes 4,5, regions c-e). In contrast, in wild-type MATa cells (Fig. 1A, lanes 2,3), the nucleosomes in this region appear to be shifted and less precisely positioned relative to that seen in MAT $\alpha$ cells (Shimizu et al. 1991). Deletion of either the ISW2 or ITC1 subunit of the Isw2 complex in MAT $\alpha$ cells results in a digestion pattern that more closely resembles that of wild-type MATa cells (Fig. 1A, lanes 6-9), consistent with previous observations (McConnell et al. 2004).

We recently determined that the $M A T \alpha$-specific transcriptional repressor, $\alpha 2 \mathrm{p}$, is required for Isw 2 to remodel chromatin at a-specific gene promoters to a repressive state (data not shown), suggesting that $\alpha 2 \mathrm{p}$ might physically recruit Isw2 to a-specific genes. We tested this prediction by ChIP of $3 \times$ Flag epitope-tagged wild-type Isw $2 p$ at the promoter of the a-specific gene STE6 in the presence or absence of $\alpha 2 \mathrm{p}$. Specifically, we compared the cross-linking of wild-type Isw2p in MAT $\alpha$ cells, in which $\alpha 2 \mathrm{p}$ binds to its operator upstream of STE6, relative to $M A T a$ cells that do not express $\alpha 2 \mathrm{p}$ and MAT $\alpha$ cells with point mutations in the STE6 promoter that disrupt $\alpha 2 \mathrm{p}$ binding $\left(\alpha 2^{-} M A T \alpha\right)$. We expected that loss of $\alpha 2 \mathrm{p}$ binding would result in a reduction in Isw2p cross-linking to the STE6 promoter. In contrast with our hypothesis, in the absence of $\alpha 2 \mathrm{p}$ binding (MATa or $\left.\alpha 2^{-} M A T \alpha\right)$, we observed only a modest decrease in Isw2p association at the $\alpha 2$ operator relative to wild-type MAT $\alpha$ (Fig. 1B, region c). We made similar observations at the promoter of the early meiotic gene, REC104 (Supplementary Fig. 1). Thus, cross-linking of wild-type Isw2p at two classes of Isw2-regulated promoters did not require the transcription factors necessary for Isw2 activity.

We next tested whether wild-type Isw2p cross-linked preferentially to its targets relative to sites of Isw2-independent chromatin structure. We examined STE6 because nucleosome mapping had revealed three distinct regions of chromatin structure at this locus. $\alpha 2 \mathrm{p}$ is required for Isw2 to remodel chromatin over the STE6 promoter (Fig. 1A, regions c-e, orange), but is dispensable for
Figure 1. Catalytically inactive Isw $2 \mathrm{p}-\mathrm{K} 215 \mathrm{R}$ is enriched at Isw2 target genes. $(A)$ Chromatin analysis reveals distinct regions of Isw2 activity upstream of STE6. Inferred nucleosome positions are marked by gray ovals. The relevant genotypes are shown along the top, and " $\mathrm{N}$ " indicates naked DNA digestion. The distance relative to the STE6 translational start site is shown to the left. The locations of the PCR products from ChIP analyses are shown to the right. (B) Graphical representation of ChIP of wild-type Isw2p and Isw2p-K215R along the STE6 locus. For each sample, ChIP of STE6 and PPA1 (loading control; see Materials and Methods) is calculated as a percentage of the input. The data are shown as a ratio of these values (fold enrichment relative to PPA1). The relevant genotypes are indicated in the upper right corner. The STE6 PCR products, labeled a-g, are diagrammed according to their position along the STE6 locus. Results of chromatin analysis for each region are summarized below.
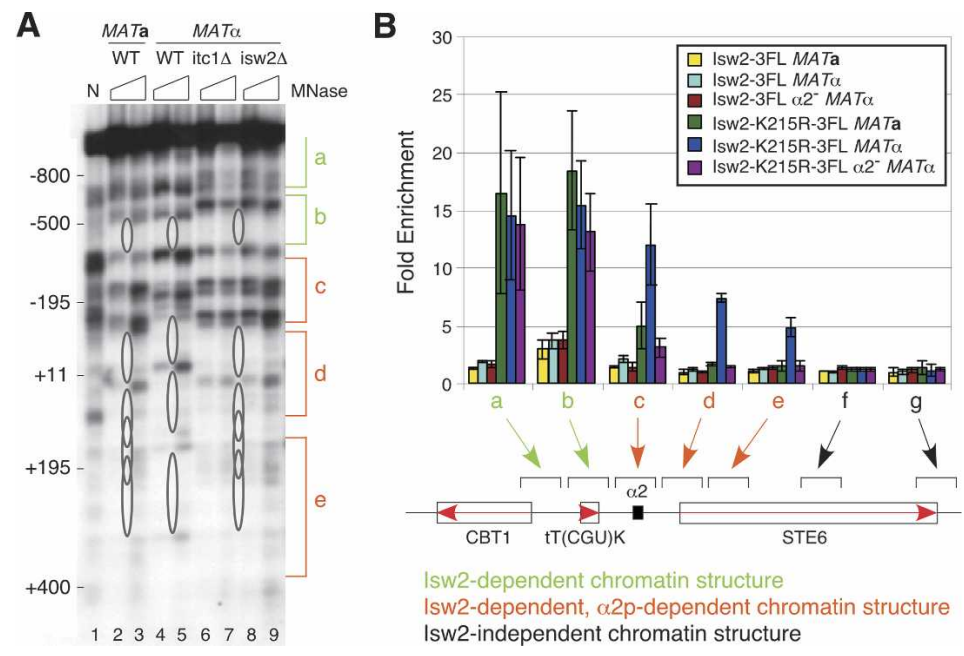
Isw2 function further upstream between the $C B T 1$ gene and the $t T(C G U) K$ tDNA (Fig. $1 \mathrm{~A}$, regions a-b, light green). We did not observe any effect of ISW2 mutation on chromatin structure over the middle and at the 3 '-end of the STE6 gene (data not shown; Fig. 1B, regions $f$ and $\mathrm{g})$. With the exception of region $b$, wild-type Isw $2 p$ crosslinks comparably to these regions despite significant differences in Isw2 activity (Fig. 1B), indicating that wildtype Isw2p is not specifically enriched at sites of Isw2 function. We have also observed similar wild-type Isw2p ChIP signals at multiple ISW2-independent loci, including ACT1 (T.G. Fazzio, M.E. Gelbart, and T. Tsukiyama, in prep.; T. Tsukiyama, unpubl.).

\section{Catalytically inactive Isw2p-K215R marks Isw2 targets}

Failure to detect specific localization of wild-type Isw2p at target promoters led us to consider two models. One is that the Isw2 complex is locally regulated solely at the level of activity, not association with chromatin. Alternatively, it is possible that Isw2 recruitment is transient and, as a result, was not detected above background in the ChIP assay. To investigate these possibilities, we used a catalytically inactive Isw2p mutant in the ChIP assay. We hypothesized that a mutation blocking chromatin remodeling might lead to persistence of a normally transient recruitment. A K215R substitution in the putative ATP-binding pocket of Isw2p reduces ATPase activity to background levels without affecting Isw2 complex integrity (Gelbart et al. 2001; Fitzgerald et al. 2004). Isw2-K215R mutants exhibit similar chromatin defects as isw2 2 mutants in vivo (T.G. Fazzio, M.E. Gelbart, and T. Tsukiyama, in prep.; T.G. Fazzio and T. Tsukiyama, unpubl.), and genes misregulated in the isw2-K215R mutant significantly overlap with those affected in the isw2 $2 \Delta$ mutant by transcriptional profiling (Fazzio et al. 2001).

We analyzed the association of Isw2p-K215R surrounding the STE6 locus by ChIP assay (Fig. 1B). Unlike wild-type Isw2p, Isw2p-K215R preferentially cross-links to Isw2 targets. At the STE6 promoter (Fig. 1B, regions c-e, orange), Isw $2 \mathrm{p}-\mathrm{K} 215 \mathrm{R}$ is enriched relative to wildtype Isw2p in MAT $\alpha$ cells, but not in the absence of $\alpha 2 p$ binding (MATa or $\alpha 2^{-} M A T \alpha$ cells). Thus, both Isw2pK215R cross-linking and Isw2 activity are $\alpha 2 \mathrm{p}$-dependent in this region. The upstream region spanning the CBT1 promoter and the $t T(C G U) K$ tDNA is also enriched by Isw2p-K215R relative to wild-type (Fig. 1B, regions a and $b$, light green), but $\alpha 2 p$ is not required for Isw2p-K215R enrichment or Isw2 function at this locus. Finally, Isw2p-K215R is not enriched relative to wildtype Isw2 $p$ in regions $f$ and $g$ (black), where chromatin structure is unaffected in the isw2 mutant.

Similar results were observed at the promoters of two early meiotic genes, INO1 and REC104 (Supplementary Fig. 1). At each locus, a two- to fourfold enrichment of Isw2p-K215R over wild-type Isw2p was observed, and this enrichment requires a functional Ume6p-binding site (URS1) at REC104. Taken together, these data sug- gest that ChIP of the catalytically inactive Isw2p-K215R mutant, but not wild-type Isw2p, is a good marker for Isw2 function.

\section{Wild-type Isw2p and Isw2p-K215R show distinct distributions across the genome}

We next sought to test whether Isw2p-K215R cross-linking is enriched at Isw2 targets on a global scale. To this end, we examined the genome-wide localization patterns of wild-type Isw2p and Isw2p-K215R. ChIP input and immunoprecipitated (IP) DNA were amplified by ligation-mediated PCR in the presence of either Cy3-dUTP or Cy5-dUTP. The differentially labeled samples were hybridized to yeast open reading frame (ORF) or intergenic microarrays, and the relative enrichment of each locus was expressed as a $\log _{2}$ ratio of IP/input. Each experiment was median-normalized and performed in triplicate with dye swap pairs (see Materials and Methods). To confirm the array results, we performed locus-specific PCR at 20 sites from the ORF and intergenic arrays (Supplementary Table 1). The array data are consistent with the PCR results, with Pearson correlation coefficients $(r)$ ranging from 0.75 to 0.98 .

A scatterplot of the average log ratios for wild-type Isw2p and Isw2p-K215R on ORF arrays is shown in Figure $2 \mathrm{~A}$. If these proteins were comparably distributed along chromatin, the data should fall along the diagonal line (green). This pattern was seen between replicate experiments, with Pearson correlation coefficients $(r)$ ranging from 0.80 to 0.94 for all pairwise comparisons (data not shown). In contrast, we observed distinct localization patterns for wild-type Isw2p and Isw2p-K215R. The majority of loci enriched by Isw2p-K215R (above the blue-dotted line) are distributed around the $Y$-axis, indicating that they are not enriched by wild-type Isw $2 p$. In
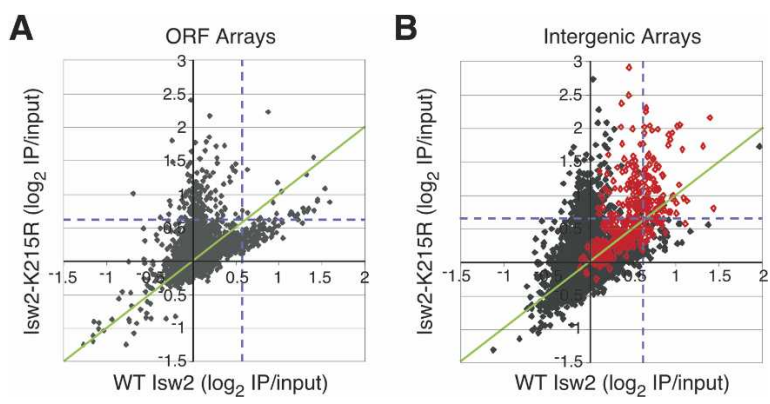

Figure 2. Wild-type and catalytically inactive Isw2p show distinct distributions along chromatin. (A) Scatterplot of Isw2p$\mathrm{K} 215 \mathrm{R}$ versus wild-type Isw2p on ORF arrays. The data are plotted as $\log _{2}$ ratios of the IP relative to the input and represent the average of three independent median-normalized experiments. The blue dotted lines indicate 1.5 -fold enrichment. The green line represents the hypothetical pattern of the data if wild-type Isw2p and Isw2p-K215R shared the same distribution along chromatin. $(B)$ Scatterplot as in $A$ on intergenic arrays. Loci adjacent to tDNAs are shown in red. The remaining intergenic spots are shown in gray. Similar patterns are seen on ORF and intergenic arrays with the exception of tDNA-adjacent spots, which are enriched by both wild-type Isw2p and Isw2p-K215R. 
general, the remaining loci fall near the diagonal. Those loci enriched by wild-type Isw2p (to the right of the bluedotted line) are also enriched, but to a lesser extent, by Isw2p-K215R, and the same loci are relatively depleted of both proteins.

A similar pattern is observed on intergenic arrays, except that there are more loci enriched by both wild-type Isw2p and Isw2p-K215R (Fig. 2B). Loci adjacent to nuclear tDNAs (Fig. 2B, in red) account for this difference between the ORF and intergenic array patterns, suggesting that some feature of these loci favors association of both wild-type Isw2p and Isw2p-K215R (discussed below).

\section{Genome-wide location analysis of Isw2p-K215R identifies Isw2 target sites}

The location analysis data sets were used to test the model that Isw2p-K215R is enriched at Isw2 targets. We analyzed the chromatin structure in wild-type and isw2 mutants at several loci enriched in the array experiments. The MNase digestion pattern indicates that nucleosomes are shifted in the isw2 mutant relative to wild type over intergenic regions, itF(GAA)G and iYPL201c, and the CSM4 ORF, loci more strongly enriched by Isw2p-K215R (Fig. 3A,B). Several other loci enriched by Isw2p-K215R in the array experiments also showed Isw2-dependent chromatin structure (Figs. 1A [regions a and b], 4E, 6C,F [below]; data not shown). In contrast, loci enriched more strongly by wild-type Isw $2 p$ than by Isw2p-K215R did not show significant changes in chromatin structure in the isw2 mutant (Fig. 3C; data not shown).
We further tested the correlation between Isw2pK215R ChIP enrichment and Isw2 function in a global manner by comparing the ChIP analyses to previously generated expression microarray data sets (Fazzio et al. 2001). Since the isw2 mutation alone has subtle effects on transcription, we used transcription data from an rpd3 background, which exhibits robust Isw2-dependent transcriptional effects (isw2 2 rpd3s vs. $r p d 3 \Delta$ ). The transcription data point for each ORF was aligned with the location data of wild-type Isw2p and Isw2p-K215R at the promoter, ORF, or 3' region. The method used to align these data sets is described further at http://www. fhcrc.org/labs/tsukiyama/supplemental-data/ isw2locationanalyses.

A significant overlap was observed between Isw2-dependent genes and genes with Isw2p-K215R enriched at their promoters (Fig. 3D). This result is consistent with the Isw2-dependent chromatin structure observed upstream of several Isw2 target genes (Goldmark et al. 2000; Fazzio et al. 2001; Kent et al. 2001). A smaller but still significant overlap was seen with Isw2p-K215R association at the 3 '-end of Isw2-dependent genes, potentially reflecting the role of Isw2 in transcriptional termination (Alen et al. 2002). Although statistically significant, the overlap between Isw2p-K215R localization and Isw2-dependent genes was most likely incomplete because (1) misregulation of some genes in the isw2 $\Delta$ $r p d 3 \Delta$ mutant may be indirect; (2) RPD3 may function in a parallel pathway with ISW2 to regulate only one class of Isw2 target genes; or (3) Isw2-dependent chromatin remodeling may regulate processes other than transcription. In contrast, there was little overlap between wildtype Isw2p localization and genes misregulated in the
Figure 3. Isw2p-K215R cross-linking marks Isw2 targets on a genome-wide scale. $(A-C)$ Nucleosome mapping at loci preferentially enriched by Isw2p-K215R $(A, B)$ or wild-type Isw2p $(C)$ in the array experiments. The relevant genotype is indicated along the top. "N" indicates naked DNA digestions. The 100-bp ladder is labeled "M.". The genomic structure of each locus is diagrammed to the right and " $\mathrm{i}$ " indicates intergenic regions. For spots that are enriched at least 1.5 -fold by either wild-type Isw2p or Isw2p-K215R, the $\log _{2}(\mathrm{IP} /$ input) for both genotypes is listed below the spot name. This value represents the average of three replicates, unless noted parenthetically. Differences in MNase accessibility between wild-type and isw2 are marked with a filled diamond or open circle, respectively. $(D)$ Enrichment of wild-type Isw2 $\mathrm{p}$ and Isw2p-K215R was compared to the expression microarray data set for isw2 $\Delta$ $r p d 3 \Delta$ versus $r p d 3 \Delta$. The expression data point for each gene was aligned with the Isw2p localization data point over the promoter region $\left(5^{\prime}\right)$, the ORF itself, or the $3^{\prime}$ intergenic region. The overlap between Isw $2 p$ enrichment and Isw2 regulation of the corresponding gene is shown with the $p$-value calculated by random sampling in parentheses. Genes that changed expression $\geq 1$.7-fold were considered Isw2-regulated (Isw2-reg) as defined previously (Fazzio et al. 2001). A 1.5-fold cutoff was used to define enrichment of wild-type Isw2p (WT en) and Isw2p-K215R (K215R en).
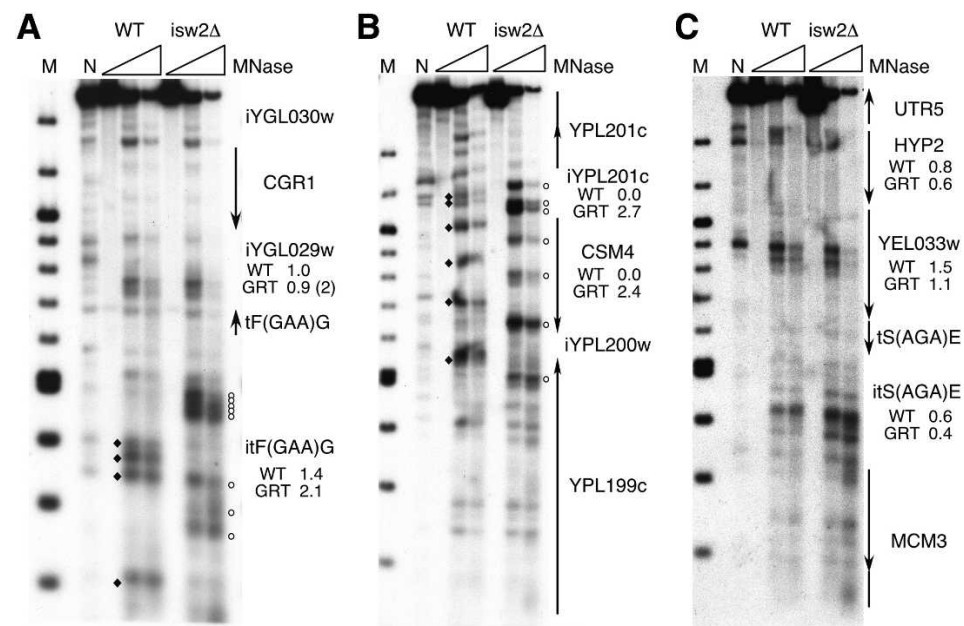

\begin{tabular}{|c|c|c|c|}
\hline & Isw2-reg. & K215R en. & overlap ( $p$-value) \\
\hline $5^{\prime}$ & 183 & 299 & $37\left(<1 \times 10^{-4}\right)$ \\
\hline ORF & 226 & 213 & $10 \quad(0.073)$ \\
\hline $3^{\prime}$ & 166 & 212 & $12 \quad(0.017)$ \\
\hline
\end{tabular}

\begin{tabular}{|cccc|}
\hline Isw2-reg. & WT en. & \multicolumn{2}{c|}{ overlap (p-value) } \\
\hline 183 & 57 & 1 & $(0.21)$ \\
\hline 226 & 115 & 0 & $(0.020)$ \\
\hline 166 & 94 & 4 & $(0.17)$ \\
\hline
\end{tabular}


A

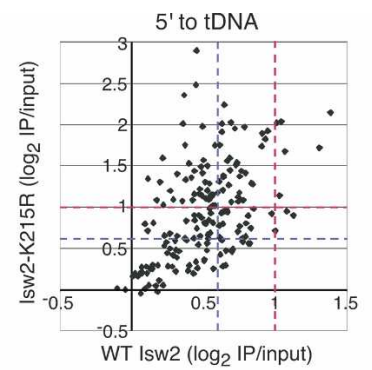

B

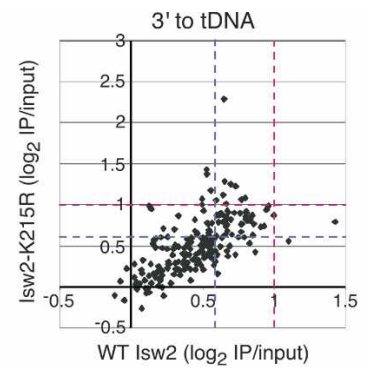

C

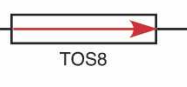

D

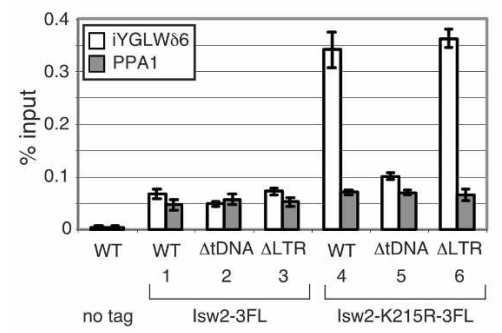

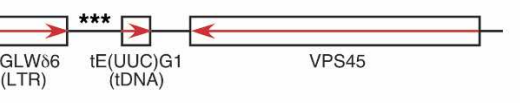

E

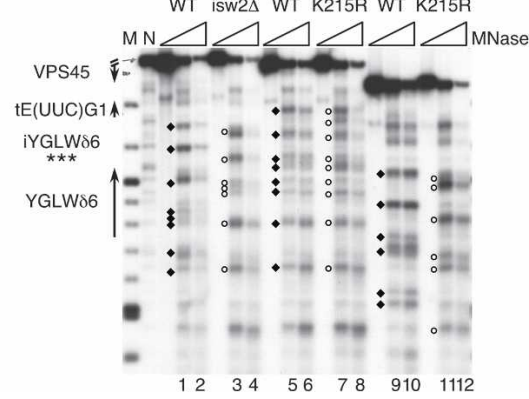

Figure 4. $t E(U U C) G 1$ recruits Isw2p-K215R and Isw2 activity to its $5^{\prime}$-flank. (A) Isw2p-K215R is preferentially enriched upstream of tDNAs. Scatterplot as in Figure 2A, except only regions upstream of tDNAs are shown. The blue and pink dotted lines indicate 1.5-fold and twofold enrichment, respectively. (B) Scatterplot as in $A$, except only regions downstream of tDNAs are shown. $(C)$ Diagram of the TOS8-VPS45 locus used for precise deletion of a tDNA or a Tyl LTR. The asterisks represent $i Y G L W \delta 6$, the intergenic locus where Isw2p-K215R was enriched in the genome-wide location analyses. $(D)$ ChIP of wild-type Isw2p and Isw2p-K215R at the TOS8-VPS45 locus. The relevant genotypes are listed along the bottom. Primers were specific for iYGLW 6 (white) or the PPA1 control (gray), and the IP signal was calculated as a percentage of the input. $(E)$ Chromatin analysis at the TOS8-VPS45 locus. Chromatin structure was analyzed at the wild-type (lanes 1-4), tDNA-deleted (lanes 5-8), and LTR-deleted (lanes 9-12) loci. ISW2 is wild type in lanes 1, 2, 5, 6, 9, and 10 and mutant in lanes $3,4,7,8,11$, and 12. No significant differences in chromatin structure were observed between isw2-K215R and isw2 2 mutants at the wild-type TOS8-VPS45 locus (data not shown). The wild-type locus is diagrammed to the left. Prominent MNase cleavage sites in the region of interest are marked with a filled diamond (wild type) or open circle (isw2). absence of ISW2. These results further support the validity of Isw2p-K215R cross-linking as a marker for Isw2 activity in vivo.

\section{Isw2p-K215R is localized upstream of $t D N A s$}

Given the results above, we reasoned that enrichment of Isw2p-K215R along the genome might reveal additional Isw2 targets in vivo. Upon analyzing the data sets, we observed enrichment of Isw2p-K215R $\geq 1$.5-fold at approximately half of the intergenic regions adjacent to nuclear tDNAs (Fig. 2B, in red). Isw2p-K215R enrichment was biased to the $5^{\prime}$-flank of tDNAs, upstream of $71 \%$ and downstream of $40 \%$ of tDNAs (Fig. $4 \mathrm{~A}, \mathrm{~B}$ ). This bias was stronger using a more stringent twofold cutoff; using this criterion, Isw2p-K215R was enriched upstream of $44 \%$ and downstream of $7 \%$ of tDNAs. The $5^{\prime}$ bias for Isw2p-K215R enrichment is of particular interest because these regions are the primary sites of integration of four classes of Ty retrotransposons in the yeast genome (Kim et al. 1998).

Ninety percent of Ty1, Ty2, Ty3, and Ty4 sequence is found within $750 \mathrm{bp}$ of a tDNA in the yeast genome, and roughly two-thirds of tDNAs are associated with Ty sequence (Kim et al. 1998). These Ty insertions are biased heavily toward the $5^{\prime}$-flank of tDNAs; of 59 Tyl insertions near pol III-transcribed genes studied by Bolton and Boeke (2003), 71\% were upstream of the tDNA. Given this proximity, it was expected that we also found increased Isw2p-K215R cross-linking adjacent to full- length Ty elements and solo long terminal repeats (LTRs). However, the presence of Ty sequence upstream of a tDNA does not appear to be a general requirement for Isw2p-K215R enrichment, since Isw2p-K215R is enriched upstream of $t T(C G U) K$ in the absence of any associated Ty sequence (Fig. 1B, regions a and b). Aside from tDNAs and Ty retrotransposons, we did not identify Isw2p-K215R enrichment associated with any other chromosomal feature or location.

tDNA-adjacent regions are the only class of loci enriched by both wild-type Isw2p and Isw2p-K215R in the array experiments (Fig. 2B). However, roughly half of the tDNA-adjacent loci enriched $\geq 1.5$-fold by Isw $2 \mathrm{p}-\mathrm{K} 215 \mathrm{R}$ are not significantly enriched by wild-type Isw $2 p$, and wild-type Isw2p does not show a pronounced bias to the 5 'flank of tDNAs (Fig. 4A,B). These results indicate that, even near tDNAs, wild-type Isw2p and Isw2pK215R often show distinct distributions. At $t T(C G U) \mathrm{K}$ and $t F(G A A) G$, Isw2p-K215R is more highly enriched than wild-type Isw2p, and nucleosome positions are altered in an Isw2-dependent manner (Figs. 1A, 3A, respectively). In contrast, wild-type Isw $2 p$ is more strongly enriched than Isw2p-K215R upstream of $t S(A G A) E$, and no significant changes in chromatin structure are observed at this locus in the isw2 mutant (Fig. 3C).

\section{A tDNA directs Isw2p-K215R enrichment and Isw2 activity}

We next investigated the mechanism underlying Isw2 targeting to tDNAs. Specifically, we tested whether 
tDNAs and/or Ty1 elements are required for enrichment of Isw2p-K215R and Isw2 activity at these loci. We generated precise deletions of the Ty1 solo LTR (YGLWS6) or the tDNA ( $t E(U U C) G 1)$ downstream of the convergently transcribed TOS8 and VPS45 genes (Fig. 4C). TFIIIC binds to the internal promoter of tDNAs and recruits TFIIIB, which, in turn, recruits pol III (for review, see Geiduschek and Kassavetis 2001). Consequently, deletion of $t E(U U C) G 1$ also removes the associated pol III regulatory sequence. We examined the effects of these deletions on Isw2p-K215R cross-linking and chromatin structure upstream of $t E(U U C) G 1$ (Fig. 4C, iYGLW 66, asterisks). The ChIP signal of Isw2p-K215R is nearly fivefold higher at $i Y G L W \delta 6$ relative to the PPA1 control locus (Fig. 4D, column 4). Deletion of the tDNA (Fig. 4D, column 5), but not the LTR (Fig. 4D, column 6), significantly reduced Isw2p-K215R cross-linking at this locus. In contrast, wild-type Isw2p is not enriched at $i Y G L W \delta 6$ relative to PPA1 (Fig. 4D, column 1), and its cross-linking is unaffected by either deletion (Fig. 4D, columns 2,3).

Nucleosomes are shifted in position in isw2 mutants relative to wild-type at this locus (Fig. 4E, lanes 1-4). Deletion of the LTR did not impair the ability of Isw2 to remodel chromatin (Fig. 4E, lanes 9-12). However, when the tDNA is deleted in an otherwise wild-type strain, the MNase digestion pattern resembles that of the isw2 mutant (Fig. 4E, cf. lanes 3,4 and 5,6). Mutation of ISW2 in this background results in only subtle changes in chromatin structure (Fig. 4E, cf. lanes 5,6 and 7,8), suggesting that the tDNA mediates the majority of Isw2 function at this locus. In contrast, the Ty1 LTR appears dispensable for Isw2 recruitment and function. The requirement of the tDNA both to enrich Isw2p-K215R and to direct Isw2 chromatin-remodeling activity to its $5^{\prime}$-flank further confirms the biological relevance of Isw2p-K215R cross-linking.

ISW2 is required for the periodic Ty1 integration pattern upstream of tDNAs

In order to investigate the biological role of Isw2 at tDNAs, we first tested whether Isw2 regulates tRNA expression. However, we were unable to detect changes in nascent tRNA transcription in the isw2 mutant by primer extension (Supplementary Fig. 2), suggesting that chromatin remodeling by Isw 2 upstream of tDNAs may be involved in regulating a process other than tRNA transcription.

Since four classes of Ty retrotransposons are found predominantly upstream of tDNAs (Kim et al. 1998), we next examined the effects of Isw2 on the regulation of Ty elements. We focused on Ty1 based on its large target window, its periodic integration pattern, and the fact that the targeting mechanism has not yet been defined. First, we measured the frequency of retrotransposition of two marked Ty1 elements in wild type and the isw2 mutant: galactose-induced retrotransposition of a plasmid-borne element (Curcio and Garfinkel 1991) and spontaneous retrotransposition of the genomic Ty1270his3-AI element (Lee et al. 1998). In both cases, the isw2 mutant did not differ significantly from wild type (data not shown).

We also looked more specifically at the ability of Isw2 to regulate the nuclear steps in the Tyl life cycle, namely, transcription and integration. Despite the ability of active tDNAs to repress the transcription of nearby Ty elements (Hull et al. 1994; Bolton and Boeke 2003), transcription of individual tDNA-proximal Ty1 retrotransposons (Morillon et al. 2002) is unaffected in the isw2 mutant (data not shown). Active tDNAs are also able to target Ty1 integration (Ji et al. 1993; Devine and Boeke 1996), and periodic Tyl integration has been demonstrated upstream of two tDNA families, with four major sites of insertion separated by roughly 80 bp (Bachman et al. 2004). The insertion pattern of a population of integration events specified by a galactose-inducible Ty1 retrotransposon (pVIT41) was mapped relative to tDNAs by PCR using primers specific to the induced Tyl element and the tDNA family of interest (Fig. 5A). A Ty1 construct containing an integrase-defective Tyl element (pNB19) was used to distinguish between integrase-mediated events and insertions occurring via other mechanisms.

In a wild-type background, we observed integrase-dependent periodic Tyl insertions at the tGly ${ }^{\mathrm{GCC}}$ tDNA
Figure 5. ISW2 is required for the periodic pattern of Ty1 integration upstream of tDNAs. (A) Schematic diagram of the integration assay. Primers specific to the galactose-induced Tyl element (black), tDNA families (blue), and specific tDNAs (orange) (Fig. 6) are shown. Insertion sites in a cell population are mapped relative to the tDNA family or to the specific tDNA by PCR. $(B)$ Agarose gel analysis of the integration assays for the 16-member tGly ${ }^{\mathrm{GCC}}$ tDNA family. " $\mathrm{M}$ " represents a $100-b p$ ladder. Strains were grown on glucose or galactose as indicated. No signal was observed for strains grown on glucose, in the absence of Tyl induction. ACT1 PCR was performed as a loading control and yielded comparable amounts of product for all samples (data not shown). (C) Graphic representation of the integration assay for pVIT41-transformed strains in $B$. The distance upstream of the tDNA family transcription start site (TSS) is plotted on the $X$-axis.
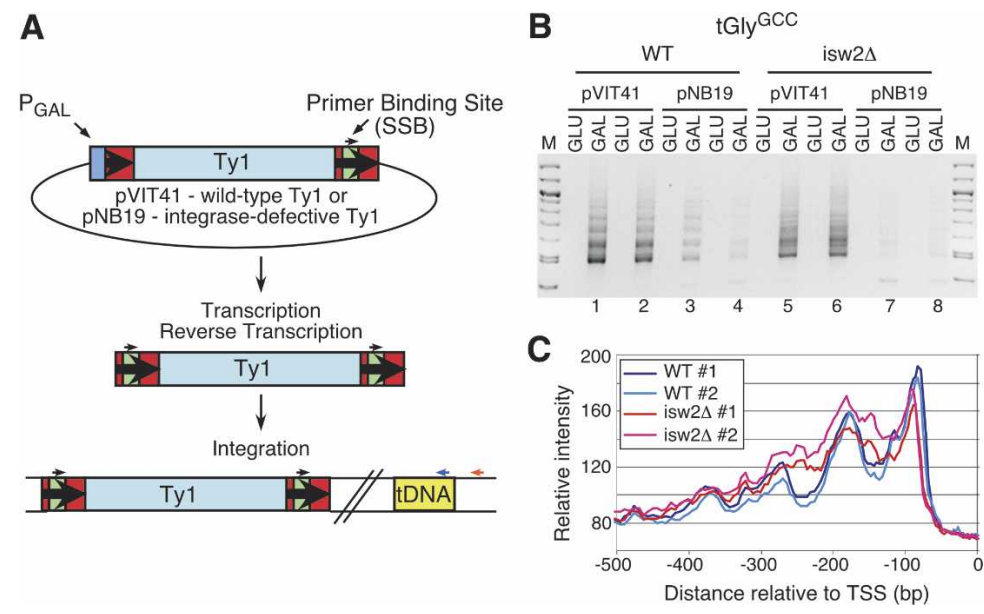
family, as described previously (Bachman et al. 2004; Fig. 5B [lanes 1-4], C). Two independent transformants for each genotype demonstrate the reproducibility of the pattern. Since the tDNA-specific primer recognizes the entire tDNA family, the pattern represents the sum of integration events upstream of 16 tGly $^{\mathrm{GCC}}$ family members. In the isw2 mutant, these tDNAs remain preferred sites of Tyl integration, but the periodic integration pattern is disrupted. Instead of the four discrete sites favored for integration in wild type, the intervening regions serve as integration targets more frequently in the isw2 mutant (Fig. 5B [lanes 5-8], C). Similar observations were made upstream of the tThr ${ }^{\text {AGU }}$ tDNA family (data not shown). Thus, Isw2 is required for appropriate Ty1 integration site selection upstream of two tDNA families.

Alterations in Ty1 integration pattern in isw2 mutants coincide with chromatin changes

To examine the correlation between Isw2-dependent chromatin remodeling and Tyl integration pattern more closely, we analyzed specific tGly ${ }^{\mathrm{GCC}}$ and $\mathrm{tThr} \mathrm{AGU}^{\mathrm{G}}$ tDNAs in the integration assay. This was done using PCR primers downstream of $t G(G C C) G 2$ and $t T(A G U) D$, tDNAs with Isw2p-K215R enriched at their $5^{\prime}$-flanks in the array experiments (Fig. 6A,D), and the signals were quantitated to facilitate the comparison between the integration pattern and chromatin structure along the locus (Fig. 6B,E). In wild type, integration at $t G(G C C) G 2$ is most favored at two sites, $\sim 90 \mathrm{bp}$ and $180 \mathrm{bp}$ upstream of the TSS (Fig. 6A [lanes 1,2], B; Bachman et al. 2004). In the isw2 mutant, preference is shifted to a pair of sites further upstream that map to the 3 '-end of YGRC 73 , a Ty4 solo LTR (Fig. 6A [lanes 5,6], B [brackets]). Chromatin analysis at this locus reveals a strong MNase cut site at the $3^{\prime}$-end of YGRC $\pi 3$ in wild-type that is absent in the isw2 mutant (Fig. 6C, diamond). Thus, Isw2 affects chromatin structure and Tyl integration pattern in the same region upstream of $t G(G C C) G 2$.

A similar result was observed at $t T(A G U) D$. Deletion of ISW2 results in an additional integration hotspot at this locus (Fig. 6D [lanes 1,2,5,6], E [brackets]). The MNase digestion pattern is consistent with the presence of a nucleosome over the 3 '-end of the Tyl solo LTR, $Y D L C \delta 1$, that is shifted in position in a subpopulation of isw2 mutant cells relative to wild type (Fig. 6F, open circles) and that overlaps with the new peak of integration. In order to ensure that these insertions arose by de novo integration and not by homologous recombination with an existing LTR, we examined the insertion pattern upon induction of an integrase-defective element. The Ty1 insertions in galactose-induced wild-type and isw2 strains reflect de novo integration events since they are absent in the integrase mutant (Fig. 6A,D, lanes 3,4,7,8).

We also found an Isw2-dependent change in chromatin structure at the $t T(A G U) N 1$ locus, not significantly enriched by wild-type Isw2p or Isw2p-K215R in the location analyses. A change in integration site selection, similar to that seen at $t T(A G U) D$, was observed overlapping the region of Isw2-dependent chromatin structure (data not shown). This result suggests that the ChIP
A

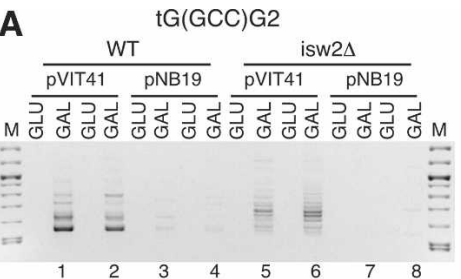

B

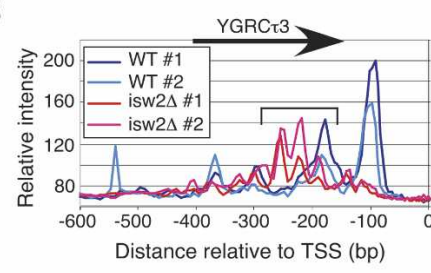

C

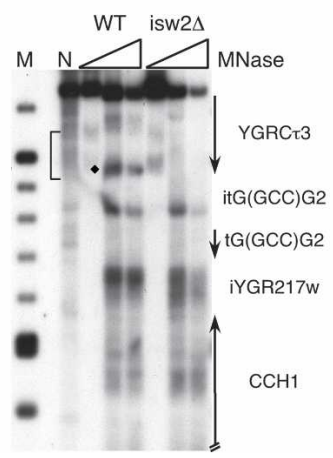

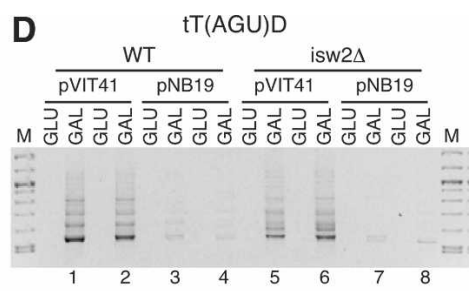

E

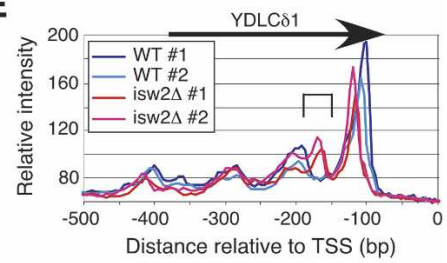

$\mathbf{F}$

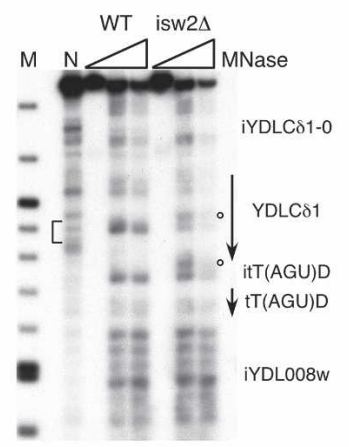

Figure 6. ISW2 affects Tyl integration pattern and chromatin structure upstream of two tDNAs. $(A, D)$ Agarose gel analysis of the integration assays at the specific tDNA copies, $t G(G C C) G 2$ and $t T(A G U) D$, as in Figure 5B. $(B, E)$ Graphic representation of the integration assays for pVIT41-transformed strains in $A$ and $D$. The positions of upstream genomic elements are marked by an arrow at the top. Differences in the integration pattern between wild type and the isw2 mutant are marked by a bracket. $(C, F)$ Chromatin analysis at $t G(G C C) G 2$ and $t T(A G U) D$. Differences in MNase accessibility between wild type and the isw2 mutant are marked with a filled diamond or open circle, respectively. Regions where the pattern of integration differs between wild type and the isw2 mutant are bracketed as in $B$ and $E$. 
analysis may underestimate the number of tDNAs targeted by Isw2 complex. The absence of a solo LTR upstream of this tDNA confirms that an LTR is not required for Isw2 to affect integration site selection.

\section{Discussion}

In this study, we first found that the localization pattern of wild-type Isw2p did not correlate with known sites of Isw2 function in vivo. In contrast, the catalytically inactive Isw2p-K215R was preferentially enriched at Isw2 targets. This result suggests that Isw2 functions transiently at its targets and that wild-type Isw2p cross-linking may not reveal functional interactions of Isw2 with chromatin (Fig. 7). Furthermore, it demonstrates that the localization pattern of the catalytically inactive Isw2pK215R mutant can be used effectively to identify Isw2 target sites in vivo. Using this method, we identified tDNAs as a new class of Isw2 targets. We determined that Isw2-dependent chromatin remodeling is required for the periodic integration of Tyl upstream of tDNAs. The regions of altered nucleosome positioning in the isw2 mutant showed changes in Ty1 integration pattern,

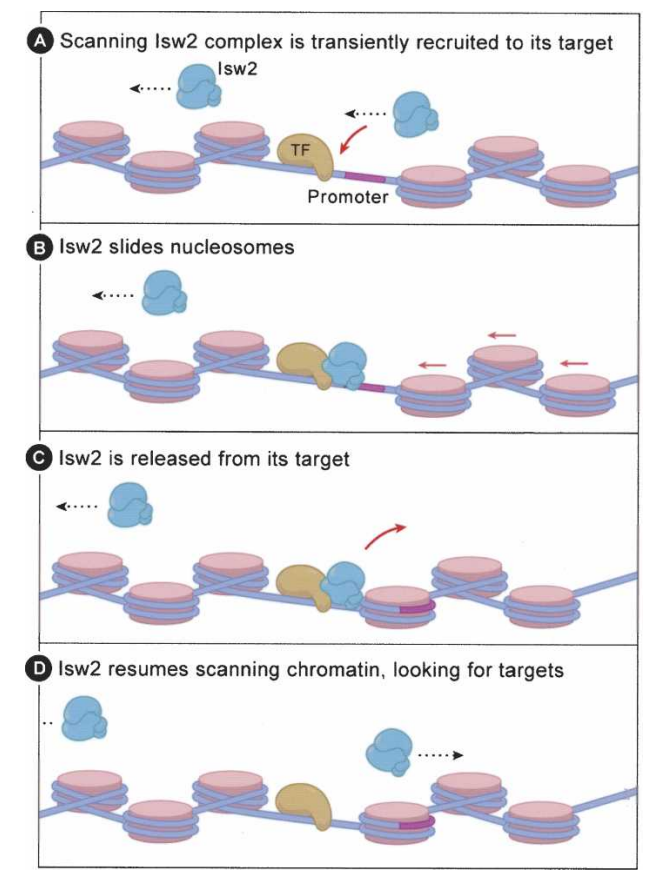

Figure 7. Model for Isw2 interactions with chromatin. We propose that Isw2 scans the genome for its targets $(A-D$, black dotted arrow). Upon identification of the appropriate target substrate $(A$, red arrow), Isw2 remodels chromatin ( $B$, red arrows) and subsequently dissociates $(C$, red arrow). The events shown in red in $A-C$ are transient and, following release from its target, Isw2 resumes its scanning mode $(D)$. The region of DNA highlighted by the purple box is to emphasize the change in nucleosome position following Isw2 action. The remodeled chromatin state is relatively stable in the absence of Isw2. In our system, the target-specific interaction is optimally detected by Isw2pK215R. demonstrating that Tyl integration is influenced by the chromatin structure upstream of tDNAs.

\section{Chromatin remodeling by Isw2 influences Ty1 integration site selection}

Isw2p-K215R localization serves as a tool to investigate the activity of an ATP-dependent chromatin-remodeling complex genome-wide. The absence of a methodology to globally map nucleosome positions in vivo previously hindered our ability to identify Isw2 targets, especially at sites where Isw2 activity does not affect transcription. We found enriched cross-linking of Isw2p-K215R upstream of many nuclear tDNAs and near Ty retroelements. Furthermore, we determined that the $t E(U U C) G 1$ tDNA was responsible for localizing Isw2p-K215R and targeting Isw2 chromatin-remodeling activity to its $5^{\prime}$ flank. These results show that tDNAs represent a new class of Isw2 targets and that Isw2 is responsible for positioning nucleosomes upstream of these genes. These findings are consistent with previous observations that transcriptionally competent tDNAs can direct nucleosome positioning in vivo (Morse et al. 1992).

Since the pol III general transcription machinery recognizes the internal promoter of tDNAs, it is likely that one or more these proteins is involved in the recruitment of Isw2. Consistent with this hypothesis, independent experiments demonstrated that an $\mathrm{N}$-terminal deletion of the Bdp1p subunit of TFIIIB altered the periodic Ty1 integration pattern (Bachman et al. 2005), similar to the present observations with an isw2 mutant. These $b d p 1$ mutants are defective in the localization of Isw2pK215R and Isw2 activity, suggesting that Bdp1p helps recruit Isw2 activity to these loci (Bachman et al. 2005).

tDNAs are the major class of Ty integration targets in the yeast genome. tDNAs are likely favorable sites for Ty integration because they are present in multiple copies, are regulated by an internal promoter, and are separated from other genes by relatively large intergenic regions, all of which reduce the likelihood of a detrimental insertion (Bolton and Boeke 2003). It is likely that Ty1 and Ty 3 targeting of tDNAs is an example of convergent evolution of a safe haven, as these two elements are phylogenetically extremely distinct. Ty1 inserts with 80-bp periodicity into a 750-bp region upstream of tDNAs (Ji et al. 1993; Devine and Boeke 1996; Bachman et al. 2004). Addition of the pol III general transcription machinery is insufficient for recapitulating Tyl targeting in vitro, suggesting that the Tyl integrase recognizes additional factors or the chromatin environment surrounding its targets (S.E. Devine and J.D. Boeke, unpubl.).

The periodic pattern of Tyl integration upstream of tDNA families suggested that the Tyl integrase might be sensitive to local chromatin structure (Bachman et al. 2004). We have demonstrated that Isw2 is required for the normal periodic Tyl integration pattern and mapped the alterations in chromatin structure that accompany changes in integration site selection in the isw2 mutant. The periodicity is also disrupted in the isw2-K215R mutant (Bachman et al. 2005), indicating that the activity of 
the Isw2 complex and not merely its physical presence affects integration. Our results show that Isw2 is responsible for creating the target chromatin structure but not for recruiting the preintegration complex to the tDNA upstream region, as tDNAs still serve as Ty1 integration "hotspots" in the isw2 mutant. Disruption of the periodic Ty1 integration pattern is specific to isw2 mutants and is not observed in 20 deletion mutants impaired in a wide variety of nuclear processes including chromatin regulation (Supplementary Table 2). The normal periodic integration was observed in strains lacking Isw1p, a homolog of Isw2p, and Rpd3p, a histone deacetylase that regulates transcription in a parallel pathway with the Isw2 complex. Although the RSC ATP-dependent chromatin-remodeling complex was also found near tDNAs in genome-wide location analyses ( $\mathrm{Ng}$ et al. 2002), mutation of the RSC subunits, HTL1 and STH1, did not disrupt the integration pattern. Neither disruption of the CAF-I chromatin assembly complex nor a wide variety of mutations in histones $\mathrm{H} 3, \mathrm{H} 4$, and the linker histone Hholp affected the normal integration periodicity. Therefore, the phenotype seen in the isw2 mutant likely reflects a specialized function of Isw2 upstream of tDNAs. The Isw2-dependent chromatin structure upstream of tDNAs may help to define tDNAs as preferred Ty1 targets redundantly with other pathways. Alternatively, Isw2 function may specifically regulate the local integration pattern. In either case, our findings provide insight into the mechanism of Tyl retrotransposon integration site selection at its preferred targets in vivo.

\section{Localization of catalytically inactive Isw2p marks} Isw2 targets in vivo

The finding that the catalytically inactive Isw2p-K215R mutant, but not wild-type Isw2p, is preferentially enriched at Isw2 targets has implications for the mechanism of Isw 2 chromatin remodeling in vivo. Our ability to detect recruitment of Isw2 complex through ChIP of Isw2p-K215R, but not wild-type Isw2p, indicates that Isw2 is recruited transiently to remodel chromatin structure. Upon substrate recognition (Fig. 7A), Isw2 remodels the local chromatin structure (Fig. 7B) and subsequently dissociates from the site (Fig. 7C). When wild-type cells are fixed for the ChIP assay, chromatin structure at the majority of Isw 2 targets is expected to be in the remodeled state represented in Figure 7D. Thus, this model provides a logical explanation for why ChIP of wild-type Isw2p does not efficiently capture its association with targets in an asynchronous cell population. Furthermore, it suggests that the remodeled chromatin state is relatively stable in the absence of Isw2. Isw2 may only be required once per cell cycle to establish proper nucleosome positioning at its targets, or it may be recruited more frequently if nucleosome positions are reset by counteracting factors. Consistent with this idea, our recent studies suggest that open chromatin structure is an important feature for recruiting Isw2 (T.G. Fazzio, M.E. Gelbart, and T. Tsukiyama, in prep.). Since Isw2 generally functions to create a closed chromatin structure (Fig.
7B), Isw2 activity would in essence destroy its own binding site, preventing subsequent recruitment events. In contrast, the inability of the catalytically inactive mutant to remodel chromatin likely leads to repeated recruitment or trapping of Isw2p-K215R at its targets (Fig. 7A), providing a wider window of opportunity to be cross-linked at these sites. Our model also predicts that a modest functional interaction of wild-type Isw $2 p$ should be detectable at loci where recruitment is robust. Consistent with this, we reproducibly observed a $30 \%$ decrease in wild-type Isw2p cross-linking at the STE6 promoter in the absence of $\alpha 2 p$ (Fig. 1B).

From chromatin and expression microarray analyses, we currently have no evidence that the general crosslinking pattern of wild-type Isw2p reflects functional interactions with chromatin. Using the ChIP assay, we believe that we detect two distinct types of chromatin interactions by Isw2 (Fig. 7). Cross-linking of wild-type Isw2p detects a basal interaction of the complex that generally does not result in chromatin remodeling (Fig. 7D). In contrast, Isw2p-K215R exhibits preferential interactions with Isw2 targets (Fig. 7A) in addition to this basal interaction with chromatin. In the absence of the transcription factor that recruits Isw2 complex (e.g., Ume $6 \mathrm{p}$ at REC104 and $\alpha 2 \mathrm{p}$ at STE6), the cross-linking of Isw2p-K215R is reduced to the basal level, that is, that observed for wild-type Isw2p. We propose that the basal interactions reflect Isw2 surveying the genome for target sites (Fig. 7D) and occur independently of ATP hydrolysis since they are also detected using the catalytically inactive mutant. It is formally possible that Isw2 function at loci enriched by wild-type Isw $2 \mathrm{p}$ was not detected by our assays. However, recent investigations have revealed that the Itc1p subunit of Isw 2 complex and a patch of basic residues on the histone $\mathrm{H} 4$ tail are required only for the target-specific interactions of Isw2pK215R (T.G. Fazzio, M.E. Gelbart, and T. Tsukiyama, in prep.). The basal levels of Isw2 cross-linking are unaffected by these mutations, suggesting that the mechanism of Isw2 association with these sites is fundamentally different from the target-specific interactions exhibited by Isw2p-K215R.

These results highlight the potential problems that can arise when ChIP of an enzyme is used as a marker for its activity. The ability to cross-link an enzyme to chromatin may not necessarily distinguish between functional and nonspecific interactions. In the case of a transiently acting factor, it is critical either to fix cells at a time when the desired reaction is occurring in a majority of the population or to stabilize the interaction. Recruitment of SWI/SNF complexes has been detected at the $\mathrm{HO}$, IFN- $\beta$, and $\alpha 1$-antitrypsin promoters during a time course of gene induction (Cosma et al. 1999; Krebs et al. 1999; Agalioti et al. 2000; Soutoglou and Talianidis 2002). It is possible that SWI/SNF complexes generally interact with their targets more stably than the Isw2 complex or alternatively that recruitment was only detectable in these studies because synchronous induction of the promoters isolated a transient window of SWI/ SNF activity. If the timing or the stability of enzyme 
recruitment is unknown, the cross-linking pattern of the wild-type enzyme must be interpreted with great caution. Catalytically inactive mutants can serve as useful tools in cases in which recruitment is unstable or transient.

\section{Materials and methods}

\section{Yeast strains}

The strains used in this study are listed in Supplementary Table 3. Point mutations in the REC104 and STE6 promoters and the ISW2 gene, and precise deletions of $t E(U U C) G 1$ and YGLW $\delta 6$ were introduced into the genome via the "pop-in pop-out" method (Rothstein 1991). Epitope-tagging with $3 \times$ Flag was performed as described (Gelbart et al. 2001).

\section{Chromatin analysis}

MNase digestions and indirect end-labeling were performed as described (Fazzio and Tsukiyama 2003), except that cells were grown in YEPD and were harvested at $\mathrm{OD}_{660}=0.7$. Zymolyase was used at $10 \mathrm{mg} / \mathrm{mL}$, and each $200-\mu \mathrm{L}$ aliquot of spheroplasts was digested with 0,25 , or $75 \mathrm{U}$ of MNase.

\section{ChIP}

ChIP was performed as described (McConnell et al. 2004), except that the fixation time was decreased to $5 \mathrm{~min}$. After reversal of cross-linking, the DNA was subjected to phenol and phenol-chloroform extractions, followed by ethanol precipitation. Western blotting indicated that similar levels of wild-type Isw2p and Isw2p-K215R are immunoprecipitated. Locus-specific PCR reactions were performed in the presence of $\left[\alpha{ }^{32} \mathrm{P}\right] \mathrm{dCTP}$, and each experiment was repeated in triplicate with three IPs from two independent chromatin samples. PPA1 was used as a loading control since it is comparably immunoprecipitated by both wild-type Isw2p and Isw2p-K215R (Fig. 4D). Chromatin structure at PPA1 is unaffected in the isw2 mutant (data not shown). Primer sequences are listed in Supplementary Table 4.

\section{Genome-wide location analysis}

For location analysis, samples were prepared as described (Ren et al. 2000), using RNase A-treated ChIP samples from MATa strains carrying $3 \times$ Flag-tagged Isw2p or Isw2p-K215R. Amplification and dye incorporation were measured by absorbance at $\mathrm{OD}_{260}, \mathrm{OD}_{550}(\mathrm{Cy} 3)$, and $\mathrm{OD}_{650}(\mathrm{Cy} 5)$ as described in the location analysis protocol version 1.2 (http://web.wi.mit.edu/ young/research/genome/protocols.html). For each array, differentially labeled input and IP DNA (20 pmol of incorporated dye) were hybridized to PCR-based spotted ORF or intergenic arrays. Three independent chromatin samples were used for each genotype with a dye swap (six arrays total per genotype). For each array, the $\log _{2}$ ratios of IP/input were median normalized. Dye swap pairs were averaged for each replicate, and the three replicates were then averaged to generate mean log ratios. Cyber-T, a Bayesian $t$-statistic derived for microarray analysis, was used to generate $p$-values (Baldi and Long 2001). We required data in all six arrays and set a lower bound threshold at 1.5-fold. This ensured $p$-values $<10^{-3}$, which remain significant when compensating for multiple testing using false discovery rate methodologies (Benjamini and Hochberg 1995). Data sets are avail- able at http://www.fhcrc.org/labs/tsukiyama/supplementaldata/isw2locationanalyses.

\section{Ty1 integration assay}

Integration assays were performed as described (Bachman et al. 2004). PCR was performed with one primer specific to the Tyl SSB (Ty1SSB1) and the other to either the tGly ${ }^{\mathrm{GCC}}$ family of tDNAs or to sequences downstream of $t G(G C C) G 2\left(5^{\prime}\right.$-TGAGA GGTGATTTTCTAGAGC-3') or $t T(A G U) D$ (5'-ATCATGCTA ACGATGACAGCG-3').

\section{Acknowledgments}

We thank members of the Tsukiyama, Boeke, Biggins, Gottschling, Tapscott, and Henikoff labs for helpful discussions, and H. Malik, S. Biggins, and members of the Tsukiyama and Boeke labs for critical reading of the manuscript. We are especially grateful to $\mathrm{S}$. Biggins for suggesting the use of a catalytically inactive Isw2p mutant in the ChIP assay. We thank Kyle Coachman and Steve Hahn for technical assistance and Bang Wong for artwork in Figure 7. We also thank D. Garfinkel and P. LeSage for their generosity with reagents and the FHCRC DNA Microarray Facility for technical support. This work was supported by NIH grant GM58465 to T.T. and NIH grant GM36481 to J.D.B. M.E.G. is supported by a Predoctoral Fellowship from HHMI. N.B. was supported in part by training grant 5T32CA09139. T.T. is a Leukemia and Lymphoma Society Scholar.

\section{References}

Agalioti, T., Lomvardas, S., Parekh, B., Yie, J., Maniatis, T., and Thanos, D. 2000. Ordered recruitment of chromatin modifying and general transcription factors to the IFN- $\beta$ promoter. Cell 103: 667-678.

Alen, C., Kent, N.A., Jones, H.S., O'Sullivan, J., Aranda, A., and Proudfoot, N.J. 2002. A role for chromatin remodeling in transcriptional termination by RNA polymerase II. Mol. Cell 10: 1441-1452.

Aye, M., Dildine, S.L., Claypool, J.A., Jourdain, S., and Sandmeyer, S.B. 2001. A truncation mutant of the 95-kilodalton subunit of transcription factor IIIC reveals asymmetry in Ty3 integration. Mol. Cell. Biol. 21: 7839-7851.

Bachman, N., Eby, Y., and Boeke, J.D. 2004. Local definition of Ty1 target preference by long terminal repeats and clustered tRNA genes. Genome Res. 14: 1232-1247.

Bachman, N., Gelbart, M.E., Tsukiyama, T., and Boeke, J.D. 2005. TFIIIB subunit Bdplp is required for periodic integration of the Tyl retrotransposon and targeting of Isw2p to S. cerevisiae tDNAs. Genes \& Dev. (this issue).

Badenhorst, P., Voas, M., Rebay, I., and Wu, C. 2002. Biological functions of the ISWI chromatin remodeling complex NURF. Genes \& Dev. 16: 3186-3198.

Baldi, P. and Long, A.D. 2001. A Bayesian framework for the analysis of microarray expression data: Regularized $t$-test and statistical inferences of gene changes. Bioinformatics 17: 509-519.

Benjamini, Y. and Hochberg, Y. 1995. Controlling the false discovery rate: A practical and powerful approach to multiple testing. I.R. Statist. Soc. B 57: 289-300.

Boeke, J.D. and Devine, S.E. 1998. Yeast retrotransposons: Finding a nice quiet neighborhood. Cell 93: 1087-1089.

Bolton, E.C. and Boeke, J.D. 2003. Transcriptional interactions between yeast tRNA genes, flanking genes and Ty elements: A genomic point of view. Genome Res. 13: 254-263. 
Bushman, F.D. 2003. Targeting survival: Integration site selection by retroviruses and LTR-retrotransposons. Cell 115: $135-138$.

Chalker, D.L. and Sandmeyer, S.B. 1992. Ty3 integrates within the region of RNA polymerase III transcription initiation. Genes \& Dev. 6: 117-128.

Cherepanov, P., Devroe, E., Silver, P.A., and Engelman, A. 2004. Identification of an evolutionarily conserved domain in human lens epithelium-derived growth factor/transcriptional co-activator p75 (LEDGF/p75) that binds HIV-1 integrase. J. Biol. Chem. 279: 48883-48892.

Collins, N., Poot, R.A., Kukimoto, I., Garcia-Jimenez, C., Dellaire, G., and Varga-Weisz, P.D. 2002. An ACF1-ISWI chromatin-remodeling complex is required for DNA replication through heterochromatin. Nat. Genet. 32: 627-632.

Cosma, M.P., Tanaka, T., and Nasmyth, K. 1999. Ordered recruitment of transcription and chromatin remodeling factors to a cell cycle- and developmentally regulated promoter. Cell 97: 299-311.

Craigie, R. 1992. Hotspots and warm spots: Integration specificity of retroelements. Trends Genet. 8: 187-190.

Curcio, M.J. and Garfinkel, D.J. 1991. Single-step selection for Tyl element retrotransposition. Proc. Natl. Acad. Sci. 88: 936-940.

Deuring, R., Fanti, L., Armstrong, J.A., Sarte, M., Papoulas, O. Prestel, M., Daubresse, G., Verardo, M., Moseley, S.L., Berloco, M., et al. 2000. The ISWI chromatin-remodeling protein is required for gene expression and the maintenance of higher order chromatin structure in vivo. Mol. Cell 5: 355365.

Devine, S.E. and Boeke, J.D. 1996. Integration of the yeast retrotransposon Tyl is targeted to regions upstream of genes transcribed by RNA polymerase III. Genes \& Dev. 10: 620-633.

Ehrenhofer-Murray, A.E. 2004. Chromatin dynamics at DNA replication, transcription and repair. Eur. J. Biochem. 271: 2335-2349.

Fazzio, T.G. and Tsukiyama, T. 2003. Chromatin remodeling in vivo: Evidence for a nucleosome sliding mechanism. Mol. Cell 12: 1333-1340.

Fazzio, T.G., Kooperberg, C., Goldmark, J.P., Neal, C., Basom, R., Delrow, J., and Tsukiyama, T. 2001. Widespread collaboration of isw2 and sin3-rpd3 chromatin remodeling complexes in transcriptional repression. Mol. Cell. Biol. 21: 6450-6460.

Fitzgerald, D.J., DeLuca, C., Berger, I., Gaillard, H., Sigrist, R., Schimmele, K., and Richmond, T.J. 2004. Reaction cycle of the yeast Isw2 chromatin remodeling complex. EMBO $\mathrm{J}$. 23: 3836-3843.

Flaus, A. and Owen-Hughes, T. 2004. Mechanisms for ATPdependent chromatin remodelling: Farewell to the tuna-can octamer? Curr. Opin. Genet. Dev. 14: 165-173.

Fyodorov, D.V., Blower, M.D., Karpen, G.H., and Kadonaga, J.T. 2004. Acf1 confers unique activities to ACF/CHRAC and promotes the formation rather than disruption of chromatin in vivo. Genes \& Dev. 18: 170-183.

Geiduschek, E.P. and Kassavetis, G.A. 2001. The RNA polymerase III transcription apparatus. J. Mol. Biol. 310: 1-26.

Gelbart, M.E., Rechsteiner, T., Richmond, T.J., and Tsukiyama, T. 2001. Interactions of Isw2 chromatin remodeling complex with nucleosomal arrays: Analyses using recombinant yeast histones and immobilized templates. Mol. Cell. Biol. 21: 2098-2106.

Goldmark, J.P., Fazzio, T.G., Estep, P.W., Church, G.M., and Tsukiyama, T. 2000. The Isw2 chromatin remodeling complex represses early meiotic genes upon recruitment by Ume6p. Cell 103: 423-433.
Hakimi, M.A., Bochar, D.A., Schmiesing, J.A., Dong, Y., Barak, O.G., Speicher, D.W., Yokomori, K., and Shiekhattar, R. 2002. A chromatin remodelling complex that loads cohesin onto human chromosomes. Nature 418: 994-998.

Huang, H., Hong, J.Y., Burck, C.L., and Liebman, S.W. 1999. Host genes that affect the target-site distribution of the yeast retrotransposon Ty1. Genetics 151: 1393-1407.

Hull, M.W., Erickson, J., Johnston, M., and Engelke, D.R. 1994. tRNA genes as transcriptional repressor elements. Mol. Cell. Biol. 14: 1266-1277.

Iida, T. and Araki, H. 2004. Noncompetitive counteractions of DNA polymerase epsilon and ISW2/yCHRAC for epigenetic inheritance of telomere position effect in Saccharomyces cerevisiae. Mol. Cell. Biol. 24: 217-227.

Ji, H., Moore, D.P., Blomberg, M.A., Braiterman, L.T., Voytas, D.F., Natsoulis, G., and Boeke, J.D. 1993. Hotspots for unselected Ty1 transposition events on yeast chromosome III are near tRNA genes and LTR sequences. Cell 73: 10071018.

Kalpana, G.V., Marmon, S., Wang, W., Crabtree, G.R., and Goff, S.P. 1994. Binding and stimulation of HIV-1 integrase by a human homolog of yeast transcription factor SNF5. Science 266: 2002-2006.

Kent, N.A., Karabetsou, N., Politis, P.K., and Mellor, J. 2001. In vivo chromatin remodeling by yeast ISWI homologs Isw $1 p$ and Isw2p. Genes \& Dev. 15: 619-626.

Kim, J.M., Vanguri, S., Boeke, J.D., Gabriel, A., and Voytas, D.F. 1998. Transposable elements and genome organization: A comprehensive survey of retrotransposons revealed by the complete Saccharomyces cerevisiae genome sequence. Genome Res. 8: 464-478.

Kirchner, J., Connolly, C.M., and Sandmeyer, S.B. 1995. Requirement of RNA polymerase III transcription factors for in vitro position-specific integration of a retroviruslike element. Science 267: 1488-1491.

Krebs, J.E., Kuo, M.H., Allis, C.D., and Peterson, C.L. 1999. Cell cycle-regulated histone acetylation required for expression of the yeast HO gene. Genes \& Dev. 13: 1412-1421.

Langst, G. and Becker, P.B. 2004. Nucleosome remodeling: One mechanism, many phenomena? Biochim. Biophys. Acta 1677: 58-63.

Lee, B.S., Lichtenstein, C.P., Faiola, B., Rinckel, L.A., Wysock, W., Curcio, M.J., and Garfinkel, D.J. 1998. Posttranslational inhibition of Tyl retrotransposition by nucleotide excision repair/transcription factor TFIIH subunits Ssl2p and Rad3p. Genetics 148: 1743-1761.

Liebman, S.W. and Newnam, G. 1993. A ubiquitin-conjugating enzyme, RAD6, affects the distribution of Ty1 retrotransposon integration positions. Genetics 133: 499-508.

Llano, M., Vanegas, M., Fregoso, O., Saenz, D., Chung, S., Peretz, M., and Poeschla, E.M. 2004. LEDGF/p75 determines cellular trafficking of diverse lentiviral but not murine oncoretroviral integrase proteins and is a component of functional lentiviral preintegration complexes. J. Virol. 78: 95249537.

Lusser, A. and Kadonaga, J.T. 2003. Chromatin remodeling by ATP-dependent molecular machines. Bioessays 25: 11921200.

Maertens, G., Cherepanov, P., Pluymers, W., Busschots, K., De Clercq, E., Debyser, Z., and Engelborghs, Y. 2003. LEDGF/ p75 is essential for nuclear and chromosomal targeting of HIV-1 integrase in human cells. J. Biol. Chem. 278: 3352833539.

McConnell, A.D., Gelbart, M.E., and Tsukiyama, T. 2004. Histone fold protein Dls1p is required for Isw2-dependent chromatin remodeling in vivo. Mol. Cell. Biol. 24: 2605-2613. 
Gelbart et al.

Morillon, A., Benard, L., Springer, M., and Lesage, P. 2002. Differential effects of chromatin and Gen 4 on the 50-fold range of expression among individual yeast Ty1 retrotransposons. Mol. Cell. Biol. 22: 2078-2088.

Morse, R.H., Roth, S.Y., and Simpson, R.T. 1992. A transcriptionally active tRNA gene interferes with nucleosome positioning in vivo. Mol. Cell. Biol. 12: 4015-4025.

Narlikar, G.J., Fan, H.Y., and Kingston, R.E. 2002. Cooperation between complexes that regulate chromatin structure and transcription. Cell 108: 475-487.

Ng, H.H., Robert, F., Young, R.A., and Struhl, K. 2002. Genomewide location and regulated recruitment of the RSC nucleosome-remodeling complex. Genes \& Dev. 16: 806-819.

Poot, R.A., Bozhenok, L., van den Berg, D.L., Steffensen, S., Ferreira, F., Grimaldi, M., Gilbert, N., Ferreira, J., and VargaWeisz, P.D. 2004. The Williams syndrome transcription factor interacts with PCNA to target chromatin remodelling by ISWI to replication foci. Nat. Cell Biol. 6: 1236-1244.

Ren, B., Robert, F., Wyrick, J.J., Aparicio, O., Jennings, E.G., Simon, I., Zeitlinger, J., Schreiber, J., Hannett, N., Kanin, E., et al. 2000. Genome-wide location and function of DNA binding proteins. Science 290: 2306-2309.

Rinckel, L.A. and Garfinkel, D.J. 1996. Influences of histone stoichiometry on the target site preference of retrotransposons Ty1 and Ty2 in Saccharomyces cerevisiae. Genetics 142: 761-776.

Rothstein, R. 1991. Targeting, disruption, replacement, and allele rescue: Integrative DNA transformation in yeast. Methods Enzymol. 194: 281-301.

Ruiz, C., Escribano, V., Morgado, E., Molina, M., and Mazon, M.J. 2003. Cell-type-dependent repression of yeast a-specific genes requires Itc $1 \mathrm{p}$, a subunit of the Isw $2 \mathrm{p}-$ Itc $1 \mathrm{p}$ chromatin remodelling complex. Microbiology 149: 341-351.

Sandmeyer, S. 2003. Integration by design. Proc. Natl. Acad. Sci. 100: 5586-5588.

Schroder, A.R., Shinn, P., Chen, H., Berry, C., Ecker, J.R., and Bushman, F. 2002. HIV-1 integration in the human genome favors active genes and local hotspots. Cell 110: 521-529.

Shimizu, M., Roth, S.Y., Szent-Gyorgyi, C., and Simpson, R.T. 1991. Nucleosomes are positioned with base pair precision adjacent to the $\alpha 2$ operator in Saccharomyces cerevisiae. EMBO J. 10: 3033-3041.

Soutoglou, E. and Talianidis, I. 2002. Coordination of PIC assembly and chromatin remodeling during differentiation-induced gene activation. Science 295: 1901-1904.

Tsukiyama, T., Palmer, J., Landel, C.C., Shiloach, J., and Wu, C. 1999. Characterization of the imitation switch subfamily of ATP-dependent chromatin-remodeling factors in Saccharomyces cerevisiae. Genes \& Dev. 13: 686-697.

Xie, W., Gai, X., Zhu, Y., Zappulla, D.C., Sternglanz, R., and Voytas, D.F. 2001. Targeting of the yeast Ty5 retrotransposon to silent chromatin is mediated by interactions between integrase and Sir4p. Mol. Cell. Biol. 21: 6606-6614.

Yasui, D., Miyano, M., Cai, S., Varga-Weisz, P., and KohwiShigematsu, T. 2002. SATB1 targets chromatin remodelling to regulate genes over long distances. Nature 419: 641-645.

Yieh, L., Kassavetis, G., Geiduschek, E.P., and Sandmeyer, S.B. 2000. The Brf and TATA-binding protein subunits of the RNA polymerase III transcription factor IIIB mediate position-specific integration of the gypsy-like element, Ty3. J. Biol. Chem. 275: 29800-29807.

Yieh, L., Hatzis, H., Kassavetis, G., and Sandmeyer, S.B. 2002. Mutational analysis of the transcription factor IIIB-DNA target of Ty3 retroelement integration. J. Biol. Chem. 277: 25920-25928.
Zhang, Z. and Reese, J.C. 2004. Ssn6-Tup1 requires the ISW2 complex to position nucleosomes in Saccharomyces cerevisiae. EMBO J. 23: 2246-2257.

Zhou, Y., Santoro, R., and Grummt, I. 2002. The chromatin remodeling complex NoRC targets HDAC1 to the ribosomal gene promoter and represses RNA polymerase I transcription. EMBO J. 21: 4632-4640.

Zhu, Y., Dai, J., Fuerst, P.G., and Voytas, D.F. 2003. Controlling integration specificity of a yeast retrotransposon. Proc. Natl. Acad. Sci. 100: 5891-5895.

Zou, S. and Voytas, D.F. 1997. Silent chromatin determines target preference of the Saccharomyces retrotransposon Ty5. Proc. Nat1. Acad. Sci. 94: 7412-7416. 


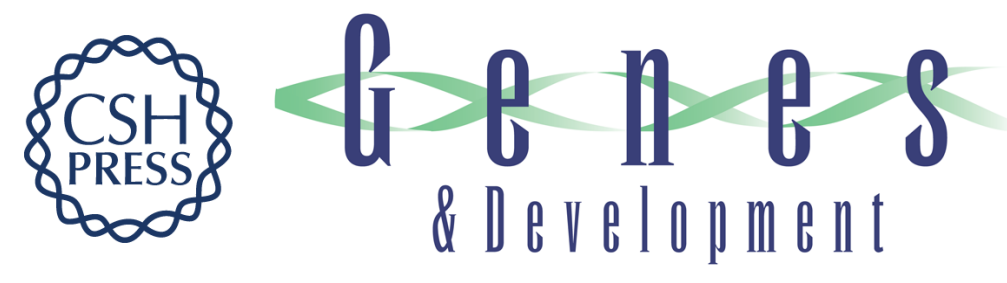

\section{Genome-wide identification of Isw2 chromatin-remodeling targets by localization of a catalytically inactive mutant}

Marnie E. Gelbart, Nurjana Bachman, Jeffrey Delrow, et al.

Genes Dev. 2005, 19:

Access the most recent version at doi:10.1101/gad.1298905

\section{Supplemental http://genesdev.cshlp.org/content/suppl/2005/04/01/19.8.942.DC1 Material}

References This article cites 66 articles, 39 of which can be accessed free at: http://genesdev.cshlp.org/content/19/8/942.full.html\#ref-list-1

\section{License}

Email Alerting

Receive free email alerts when new articles cite this article - sign up in the box at the top Service

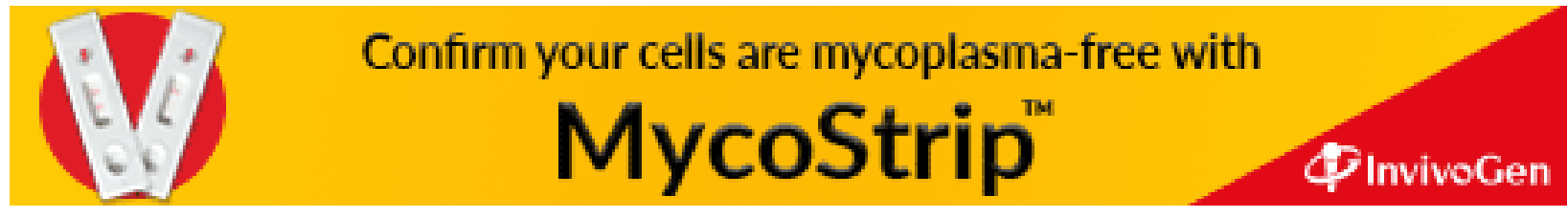

